
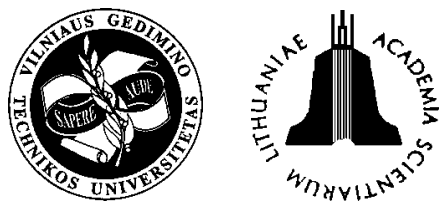

\title{
INVESTIGATION OF DYNAMIC MODELS OF INDEPENDENTLY ROTATING WHEELS OF WAGONS
}

\author{
Gintautas Bureika $^{1}$, Leonas Povilas Lingaitis ${ }^{2}, \check{S ̆ a r u ̄ n a s ~ M i k a l i u ̄ n a s ~}^{3}$ \\ Dept of Railway Transport, Vilnius Gediminas Technical University, \\ J. Basanavičiaus g. 28, LT-03224 Vilnius-09, Lithuania
}

Received 2003-09-09; accepted 2004-01-15

\begin{abstract}
The article deals with the modelling of independently rotating wheels (IRW) systems for wagons. The research work of the intensity of rolling stock wheels rims and flanges is observed. The main performance objectives of IRW are to reduce winding of wheel-sets, to enable railway rolling stocks to achieve higher speeds, to decrease wear of rail and wheel rolling surface, to improve the performance on railway curves and to reduce lateral displacement on rails. IRW with profiled tread are examined and the mathematical model of these IRW is created. Finally, the peculiarities of the stability and wearing characteristics of such IRW systems are presented.
\end{abstract}

Keywords: independently rotation wheels system, wearing characteristics, modelling of truck motion, lateral displace-

ment of wheel-set, longitudinal displacement of wheel-set.

\section{Introduction}

An ordinary wheel-set in which two wheels are mounted rigidly on an axle starts to wind (lateral oscillation) at high speeds and causes instability and wear of both wheels and rail. The intensity of traction rolling stock wheels rims and wheels flanges abrasion were investigated by many researchers, for example [1-3]. The dynamic processes in wheel/ rail contact area are discussed in some papers of the recent years [4-6]. The ultimate cause of a winding motion is in the coupling between the lateral and yaw motion of the wheel-set and this coupling is brought about by the rigid connection of the axle with tapered wheels. Researchers are attempting to solve this problem by decoupling the wheels and making them rotate independent of each other - a concept known as Independently Rotating Wheel System (IRW). The performance objectives of rail wagons equipped with IRW are: the first, to reduce severe winding present in railway wagons with conventional wheels; this will enable the rail wagons to achieve higher speeds; the second, to reduce wear of rail and of the wheel tread and flange, as well as to lower energy dissipation; the third, to improve the performance in track curves by virtually eliminating longitudinal components of creep real; the last, to reduce lateral forces on rails.

\footnotetext{
${ }^{1}$ gintautas.bureika@ti.vtu.lt

2 leonas1@ti.vtu.lt

3 smikal@ti.vtu.lt
}

Extensive performance tests were also conducted by other reseachers $[7,8]$. The main observations were the following:

1) lateral vibration in the car with IRW was less - a reduction of $60-80 \%$ on both straight and curved track;

2) sinuous motion is eliminated but there is a tendency for one of the wheel flanges to be in continuous contact with the side of the rail head;

3) no noticeable effect on wheel wear and power consumption compared with ordinary wheel-sets;

4) lateral thrust on rails is less, particularly when negotiating sharp track curves.

\section{Ground requirements}

These experiments served to reveal the basic problem with IRW system. Since the rigid connection of the wheel axle is removed, guidance in a truck using IRW is reduced only to the gravitational restoring force of the coned wheels bounded by the flanges and a small lateral force could make it run continuously against one rail, thus increasing the possibility for derailment. Hence IRW system does not have guidance capability, some method of guidance has to be provided. Among the research efforts we have discussed are those, which have centred on providing the necessary guidance to the wagons equipped with IRW.

Researchers attempted several methods to provide the guidance:

1. IRW with profiled tread - the conical tread is 
modified to a profile so as to generate a gravitational restoring force.

2. IRW with cylindrical tread - in this case a special guidance mechanism has to be provided for the system.

3. IRW with partial coupling - a partial coupling could be provided in the axle through a torsion clutch or a damper coupler.

\section{Independent rotation of wheels with profiled tread}

In a railway wheel-set, coning of the treads was introduced to prevent the constant rubbing of one or the other flange against the rail which would occur with a cylindrical tread. However, the customary coning of 1 in 20 or 1 in 40 was found quite ineffective for ordinary wheels because of the flange guidance in curves. This lead to large flange and rail wear. Hence worn profiles have been adopted which improve guidance in curves. Such a non-linear high conicity profile could be used on independent wheels so that the gravitational restoring force would provide the necessary centring effect on the wheel-sets.

For analytical prediction to be realistic and correct, IRW system has to be modelled taking into account the additional degree-of-freedom (d. o. f.) due to independent rotation of wheels.

\section{Modelling of independent rotating wheels}

An IRW configuration can be modelled similar to a ordinary wheel-set where each wheel experiences independent rotation. Various d. $\mathrm{o}$. f. for a wheel-set model is lateral, longitudinal, and vertical, yaw, roll and spin. In ordinary wheel-set dynamic study only the lateral and yaw modes are considered to describe the motion of the wheelset for small oscillations about its equilibrium position. In the case of IRW model, additionally spin d. o. f. has to be introduced to describe the motion of the system.

The important factor in the calculation of creep ages for IRW is to consider the effect of wheel rotational speeds on longitudinal and lateral creep age terms $[8,9]$. Referring to Fig 1 , let $\theta_{l, r}$ is individual rotational velocity of left and right wheels.

In small intervals of time $d t$ in seconds, the displacements at the wheel contact points are actual and due the rolling.

Components of actual displacement are:

1. Longitudinal component is $v d t \pm a d \psi$ for the left and the right wheels, where $\psi$ is rotation round an axle $\mathrm{Z}$;

2. Lateral component is $d y$.

Components of rolling displacement are:

1. Longitudinal:

$$
r \text { 'dq; }
$$

where $r^{\prime}=r_{o} \pm \lambda y$.

\section{Lateral: $r^{\prime} d \theta \psi$.}

where $\theta$ - rotation round an axle Y.

Now creep $g$, which is the ratio of difference between actual displacement and displacement due to rolling to the forward displacement, longitudinal creep can be obtained:

$$
y_{l, r}=\frac{v d t \pm a d \psi-r^{\prime} d \theta}{v d t} .
$$

By substituting $\dot{\theta}=\omega+\dot{\phi}$ and $\omega=v / r_{0}$ we have:

$$
\begin{aligned}
& \gamma_{l}=-(a / v) \dot{\psi}-\left(r_{0} / v\right) \dot{\phi}_{l}-\left(\lambda / r_{0}\right) y \\
& \gamma_{r}=-(a / v) \dot{\psi}-\left(r_{0} / v\right) \dot{\phi}_{r}-\left(\lambda r_{0}\right) y .
\end{aligned}
$$

Lateral creep is:

$\gamma=\left(d y-r^{\prime} d \theta \psi\right) / v d t=(\dot{y} / v)-\psi$.

The tangential contact force due to creep is:

$F_{c}=-f \gamma$.

Wheel-set lateral force due to creep is:

$$
F c y=-2 f(\dot{y} / v-\psi) \text {. }
$$

Wheel-set yawing moment due to creep can be attained as:

$$
\begin{aligned}
& Y_{c}=-\left(2 f a^{2} / v\right) \dot{\psi}-\left(2 f a \lambda / r_{0}\right) y- \\
& \left(2 f a r_{0} / v\right)\left(\dot{\phi}_{l}-\dot{\phi}_{r} / 2\right) .
\end{aligned}
$$

By defining $\phi=\left(\phi_{l}-\phi_{r}\right) / 2$, we gain:

$$
Y_{c}=-\left(2 f a^{2} / v\right) \dot{\psi}-\left(2 f a \lambda r_{0}\right) y-\left(2 f a r_{0} / v\right) \dot{\phi} \text {. }
$$

Individual wheel pitching moments are:

$$
\left(P_{c}\right)_{l, r}=f r_{0}\left((-a / v) \psi \pm\left(\lambda y / r_{0}\right)\right)-\left(r_{0} / v\right) \dot{\phi}_{l, r} .
$$

Now defining $P_{c}=\left(P_{c}\right)_{l}-\left(P_{c}\right)_{r}$, we gain:

$$
\begin{aligned}
& P_{c}=-\left(2 f r_{0} a / v\right) \dot{\psi}-f \lambda y-\left(f r_{0}^{2} / v\right) \dot{\phi} . \\
& P_{c}=-\left(2 f r_{0} a / v\right) \dot{\psi}-f \lambda y-\left(f r_{0}^{2} / v\right) \dot{\phi} .
\end{aligned}
$$

Equations (8), (9) and (10) could be incorporated into equation of motion of IRW mathematical model. So we can gain results for analysis and prediction of these systems behaviour in various conditions.

\section{Independent rotation wheels with a concave con- tour profile}

For analysis, an IRW model with concave contour profile is shown in Fig 1.

The equation of motion for the lateral model is:

$$
\begin{aligned}
& \left(M_{\omega}+a_{1}^{2} I_{2}\right) \ddot{y}-a_{1}^{2} I_{2} \ddot{y}_{1}+a_{1} I_{2}\left(1+a_{1} r_{0}\right) \ddot{\theta}_{1}+ \\
& 2 \frac{f}{v}\left(\frac{a-\rho \sin \delta}{a \cos \delta-c \sin \delta_{0}}\right)^{2}\left(\dot{y}_{2}-\dot{y}_{1}+r_{0} \dot{\theta}_{1}\right)+
\end{aligned}
$$




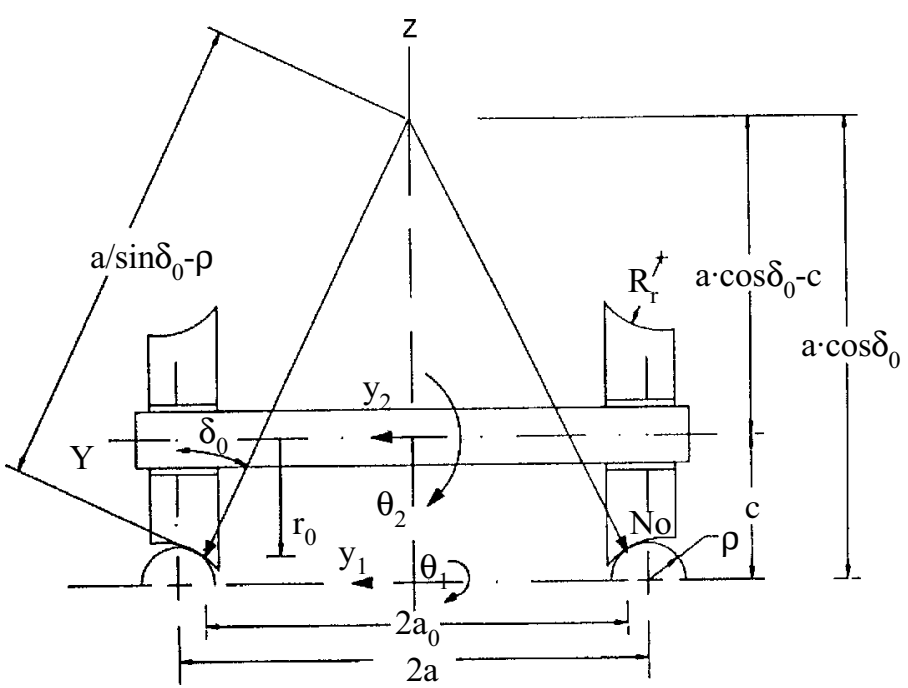

Fig 1. Physical model of independently rotating railway wheels

$$
\begin{aligned}
& 2 \frac{N_{0}}{\Delta R}\left(\frac{a^{2}+\Delta R \sin \delta_{0}\left(a \cos \delta_{0}-c \sin \delta_{0} \cos \delta_{0}+a\right)}{\left(a \cos \delta_{0}-c \sin \delta_{0}\right)^{2}}\right) . \\
& x\left(y_{2}-y_{1}+r_{0} \theta_{1}\right)+2 N_{0} \cos \delta_{0} \theta_{1}=F+a_{1} M ; \quad(11)
\end{aligned}
$$

where: $a_{1}=\left(a \cos d_{0}-c\right)^{-1}$;

$\Delta R=R_{w}-\rho-$ the difference between the radius of wheel profile $R_{w}$ and the railhead radius $\rho$.

For infinitesimal values of the contact angle $\delta_{0}$ and $c / a, \rho / a \leq 1$, the equation (11) simplifies to:

$$
\begin{aligned}
& \left(m_{\omega}+a_{1}^{2} I_{2}\right) \ddot{y}_{2}+a_{1}^{2} I_{2} \ddot{y}_{1}+a_{1}\left(1+a_{1} r_{0}\right) I_{2} \theta_{1}+ \\
& \frac{2 f}{v}\left(\dot{y}_{2}-\dot{y}_{1}+r_{0} \dot{\theta}_{1}\right)+2 N_{0}\left(\frac{1}{\Delta R}+\frac{2 \delta_{0}}{a}\right) \\
& \left(y_{2}-y_{1}+r_{0} \theta_{1}\right)+2 N_{0} \theta_{1}=F+a_{1} M
\end{aligned}
$$

where: $a_{1}=\delta_{0} / a$.

The effective lateral stiffness $K$ caused due to contact angle $\delta_{0}$ and radii difference $\Delta R$ could be written as:

$$
K=2 N\left(\frac{1}{\Delta R}+\frac{2 \delta_{0}}{a}\right) .
$$

It is seen, that positive values of contact angle $\delta_{0}$ increase the lateral stiffness and negative values of $\delta_{0}$ decrease it.

\section{Curving behaviour of IRW with non-linear conical profile}

The calculation of wheel-rail wear would be a good indication of the curving behaviour of a particular wagon. For the case of a four-wheel wagon with a flexible primary yaw suspension, the total wear $t$ (summed over all four wheels of the wagon) is shown in Fig 2 for a range of track curve radii and for both conventional and independently rotating wheels.

According to Fig 2 on shallow track curves IRW has greatly increased wear and on sharper curves the effect is less pronounced, but the wear is still bigger. As it is shown in Fig 3 for a truck with a stiff plan view primary suspension, the wear at the leading outer wheel of the truck (platform car) is virtually unaffected by the introduction of IRW. Otherwise, summing over the whole truck shows a reduction in the total wear $t$, particularly on sharper curves.

\section{Conclusions}

1. The theoretical and experimental results show that independently rotating wheel systems provided with suitable guidance will prove to be far more superior to ordinary rigid axle wheel-sets.

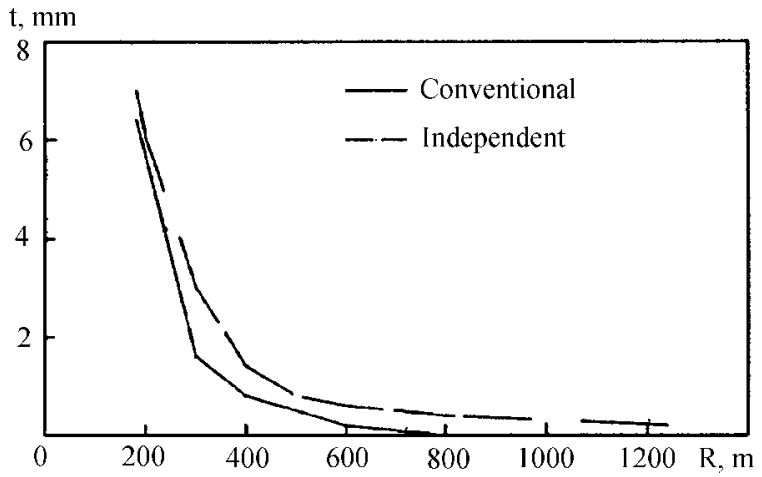

Fig 2. Total wheel wearing of 4 wheels wagon with a flexible yaw suspension 


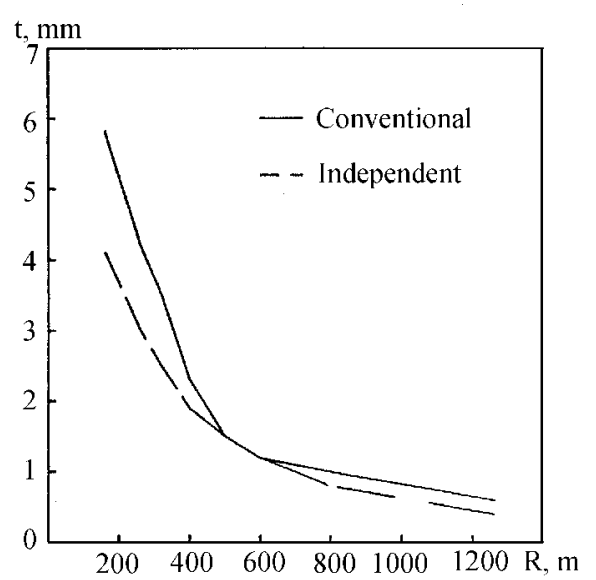

Fig 3. Total wheel wearing of truck with a stiff frame

2. For the case of a four-wheel wagon with a flexible primary yaw suspension on shallow track curves IRW has greatly increased wear and on sharper curves the effect is less pronounced, but the wear is still bigger (see Fig 2).

3. For a truck with a stiff plan suspension the wear at the leading outer wheel of the truck is virtually unaffected by the introduction of IRW, but summing over the whole truck shows a reduction in the wear, particularly on sharper curves (see Fig 3).

4. The positive values of contact angle $\delta_{0}$ increase the lateral stiffness $K$ and negative values decrease it (see Formula 13).

5. It is necessary to investigate the effects of different wheel profiles in view to improve the self-centring behaviour of IRW by optimising a wheel profile for this application.

\section{Reference}

1. Mikaliūnas, Š.; Lingaitis, L. P.; Subačius, R. Analysis of Locomotive Wheel Sets Wearing. Transport, Vol XVII, No 1, Vilnius: Technika, 2002, p. 3-7.

2. Updhay, R. Decrease of wheel and rail wear. International Railway Journal. 2000, No 7, p. 33-34. ISSN: 0744-5326.

3. Zabory, I. Prediction of wheel/ rail profile wear. Vehicle Systems Dynamics. 1997, Vol 28. Issue 2/3, p. 221-359. ISSN:0042-3114.

4. Bureika, G.; Mikaliūnas, Š. Peculiarities of traction forces in wheel/ rail contact area. Transport, Vol XVII, No 1, Vilnius: Technika, 2002, p. 8-14.

5. Bureika, G.; Subačius, R. Mathematical model of dynamic interaction between wheel-set and rail track. Transport, Vol XVII, No 2, Vilnius: Technika, 2002, p. 46-51.

6. Andersson, C.; Oscarsson, J. Dynamics train/ track interaction including state-dependent track properties and flexible vehicle components. Vehicle systems dynamics. 1999, Vol 33. Issue S, p. 47-58. ISSN: 0042-3114.

7. Dupikati, R. V.; Swamy, S. Narayana; Osman, M. O. M. Independently Rotating Systems for Railway Vehicles - A state of the Art Rewiew. Vehicle Systems Dynamics. 1992, Vol 21, No 5, p. 297-330. ISSN:0042-3114.

8. Goodall, R.; Li, H. Solid axle and independently-rotating railway wheel-sets. A control engineering assessment of stability. Vehicle System Dynamics. 2000, Vol 33, Issue 1, p. 57-67. ISSN:0042-3114. 